\title{
Teaching Fraunhofer diffraction via experimental and simulated images in the laboratory
}

Alba Peinado, Josep Vidal, Juan Carlos Escalera, Angel Lizana, Juan Campos, et al.

Alba Peinado, Josep Vidal, Juan Carlos Escalera, Angel Lizana, Juan Campos, Maria Yzuel, "Teaching Fraunhofer diffraction via experimental and simulated images in the laboratory," Proc. SPIE 8481, Optics Education and Outreach II, 84810D (15 October 2012); doi: 10.1117/12.943241

SPIE Event: SPIE Optical Engineering + Applications, 2012, San Diego, California, United States 


\title{
Teaching Fraunhofer diffraction via experimental and simulated images in the laboratory
}

\author{
Alba Peinado*a , Josep Vidal ${ }^{\mathrm{a}, \mathrm{b}}$, Juan Carlos Escalera ${ }^{\mathrm{a}}$, Angel Lizana ${ }^{\mathrm{a}}$, Juan Campos ${ }^{\mathrm{a}}$, and María \\ Yzuel $^{\mathrm{a}}$ \\ ${ }^{a}$ Department of Physics, Universitat Autònoma de Barcelona, Bellaterra, SPAIN 08193;
${ }^{b}$ ALBA Synchrotron Light Source Facility, Cerdanyola del Vallès, SPAIN 08290
}

\begin{abstract}
Diffraction is an important phenomenon introduced to Physics university students in a subject of Fundamentals of Optics. In addition, in the Physics Degree syllabus of the Universitat Autònoma de Barcelona, there is an elective subject in Applied Optics. In this subject, diverse diffraction concepts are discussed in-depth from different points of view: theory, experiments in the laboratory and computing exercises. In this work, we have focused on the process of teaching Fraunhofer diffraction through laboratory training.

Our approach involves students working in small groups. They visualize and acquire some important diffraction patterns with a CCD camera, such as those produced by a slit, a circular aperture or a grating. First, each group calibrates the CCD camera, that is to say, they obtain the relation between the distances in the diffraction plane in millimeters and in the computer screen in pixels. Afterwards, they measure the significant distances in the diffraction patterns and using the appropriate diffraction formalism, they calculate the size of the analyzed apertures. Concomitantly, students grasp the convolution theorem in the Fourier domain by analyzing the diffraction of 2-D gratings of elemental apertures.

Finally, the learners use a specific software to simulate diffraction patterns of different apertures. They can control several parameters: shape, size and number of apertures, 1-D or 2-D gratings, wavelength, focal lens or pixel size. Therefore, the program allows them to reproduce the images obtained experimentally, and generate others by changing certain parameters. This software has been created in our research group, and it is freely distributed to the students in order to help their learning of diffraction.

We have observed that these hands on experiments help students to consolidate their theoretical knowledge of diffraction in a pedagogical and stimulating learning process.
\end{abstract}

Keywords: Fraunhofer diffraction, convolution theorem, diffraction simulation, hands on experiment.

\section{INTRODUCTION}

The Physics degree at the Universitat Autònoma de Barcelona has a course of Fundamental of Optics in the third year. This subject covers topics like Geometrical Optics, Wave Optics theory (polarization, interference, diffraction, etc.) and Applied Optics (Optical Instruments) [1,2]. This is complemented with a laboratory in General Optics. Since this is a general panoramic course, diffraction theory lectures lack in mathematically thoroughness based on Fourier Transform and experimental demonstrations. In order to complement that course, an elective course in the fourth year is given, called Applied Optics. There, students gain a solid mathematical base in diffraction [3-4], as well as they learn about image processing [5] and holography [3, 6, 7]. Also, there are other elective courses like Quantum Optics and Introduction to Photonics.

*Alba.Peinado@uab.es; phone +34 935868 029; fax +34 935812155

Optics Education and Outreach II, edited by G. Groot Gregory, Proc. of SPIE Vol. 8481

84810D · (C) 2012 SPIE · CCC code: 0277-786/12/\$18 · doi: 10.1117/12.943241 
The course on Applied Optics is based on 30 hours of theory lectures plus the resolution of exercises like home work, 9 hours of laboratory work and 7 hours of computing simulations. Therefore, students learn diffraction theory from three points of view: theory, experimental work and computing exercises. The laboratory training is distributed in three sessions [8] which are focused in diffraction of Fraunhofer, coherent spatial frequency optical filtering and holography. In that way, experimental understanding consolidates the theoretical knowledge learnt at class.

Students come to the laboratory in small groups (maximum 4 people). Therefore, the laboratory teaching assistant can focus his explanation to a reduced number of students. This methodology favors the teacher-student interaction and a better understanding of the subject. Before coming to the session, students receive a guideline about the experiment. So, they will come to the laboratory with the main concepts and an overview of the experiment, leading to carry out the experiment much fluently. Each session lab lasts 3 hours, where students are active participants on the hands-on tasks.

In this work, we explain the process of teaching Fraunhofer diffraction in order to train undergraduate students by means of experimental and simulated work. In section 2, we introduce the experimental setup to visualize the diffraction patterns and the experimental methodology conducted in the laboratory. Section 3 explains the software used to acquire the images, to process and analyze them, as well as a simulation module to reproduce a large variety of diffraction patterns. Section 4 includes some results obtained by the students, based on experimental and simulated work. Section 5 includes the evaluation method for this course and finally, section 6 summarizes the main ideas of this work.

\section{EXPERIMENTAL METHODOLOGY}

\subsection{Setup}

The experimental setup used to implement the Fraunhofer diffraction is shown in Figure 1. It is illuminated by a He-Ne laser $(633 \mathrm{~nm})$. The intensity can be regulated by a variable neutral density filter. Then, the light beam is filtered by a spatial filter and collimated by the first lens. Then, a circular aperture selects the diameter size of the collimated beam. After that, the light beam is diffracted by the object or the aperture analyzed. Finally, by using a second lens, we obtain the Fraunhofer diffraction in the focal plane of that lens. There, the intensity pattern is captured by a CCD camera and then, analyzed by specific software in the computer.

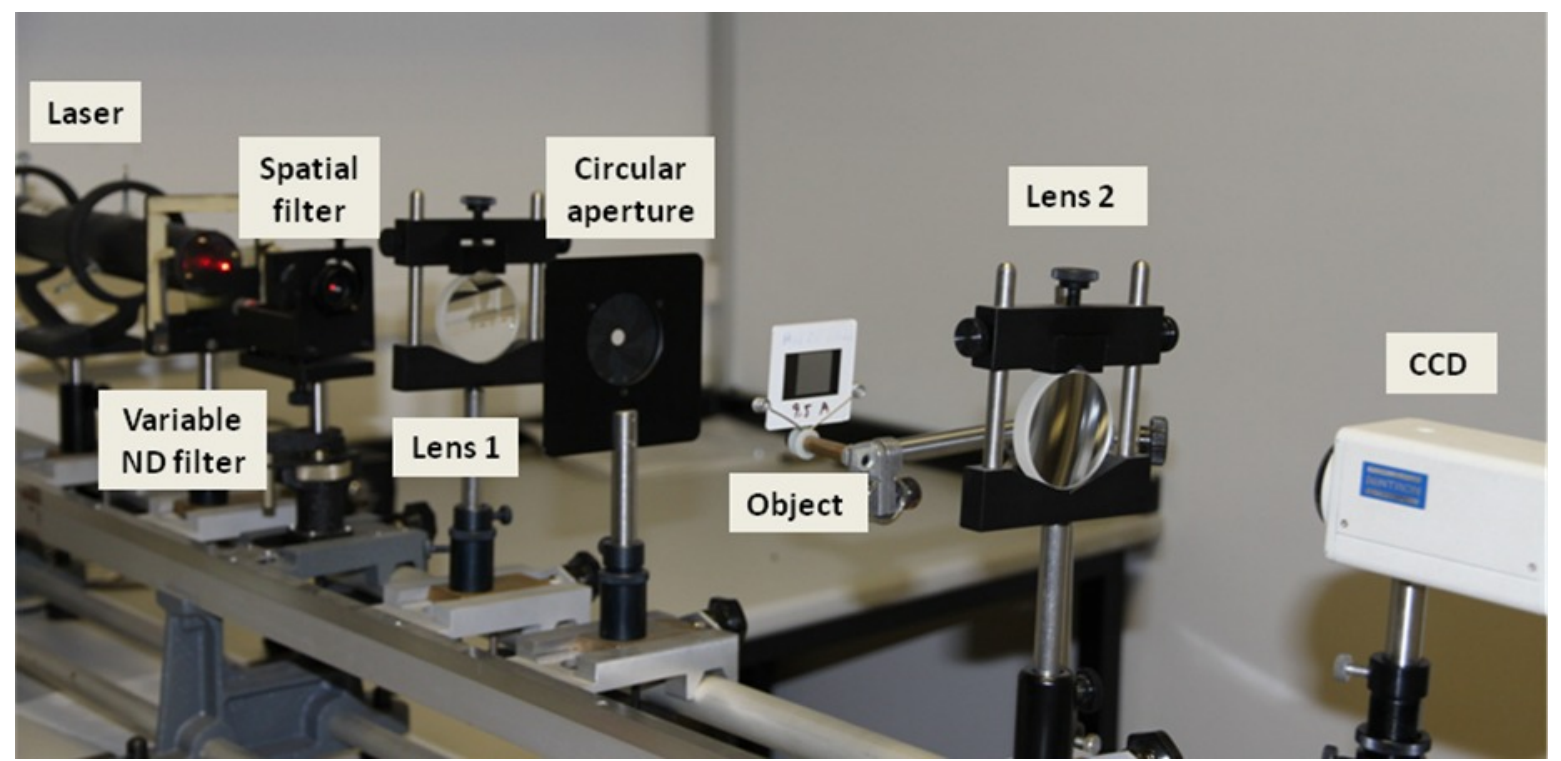

Figure 1. Experimental setup to visualize the Fraunhofer diffraction: Laser, variable neutral density filter, spatial filter, first lens, circular diaphragm, aperture to be analyzed, second lens and finally the screen or the CCD detector.

\subsection{Calibration of the camera}

First of all, students have to calibrate the CCD camera, in order to determine the relation between the distance in $\mathrm{mm}$ in the focal plane and in pixels in the digital image acquired by the camera. With that purpose, they analyze the diffraction of 1-D grating by using two different methods. First, they replace the camera by a micrometer grid eyepiece. Then, they 
visualize the diffraction orders and they measure the distance between maximums of intensity. Second, placing again the CCD camera in the focal plane, they acquire an image of the diffraction pattern, as the one showed in Figure 2. By using specific software (see section 3), they can measure the distance between maxima in pixels in that image. And finally, they can obtain the conversion factor in pixels $/ \mathrm{mm}$.

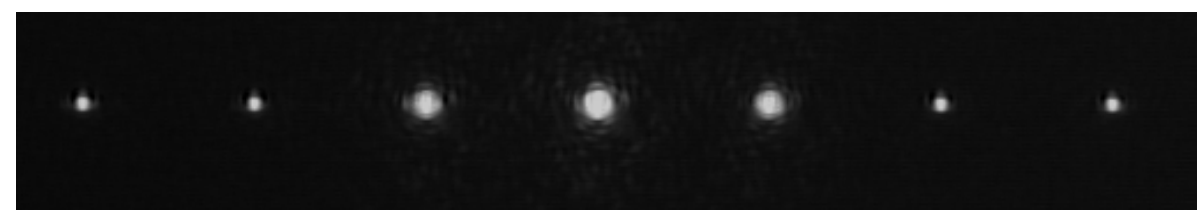

Figure 2: Diffraction of the one dimension grating used to calibrate the CCD camera

\subsection{Measurement procedure}

Different apertures will be analyzed in order to cover the diffraction patterns of the basic apertures. The list includes a slit, double slit, rectangular, square, circular and triangular aperture, double slit and, diffraction gratings in one dimension and in two dimensions (where the apertures are basic apertures).

Students will discuss with the laboratory teacher the diffraction pattern that they are expecting from each aperture based on their previous knowledge. Also, they will understand how the mathematic formula from the literature [1-4] describes a distribution of intensity in two-dimensions. Then, they will put the object in the experimental set-up and produce the diffraction pattern, allowing consolidating the concepts. The intensity pattern will be acquired by the camera. Then, measuring the pixels in the image with the specific software, using the conversion factor between pixels and millimeters, and knowing the focal length of the second lens, learners are able to determine the relevant dimensions of the aperture analyzed.

\section{SOFTWARE}

The Fraunhofer diffraction software controls the frame grabber of the camera, allowing changing the settings of the camera, such as the area of acquisition and the origin of the image. Then, the student can take a picture or make a video of the diffraction pattern. After that, the image can be processed by the program. In particular, it allows changing the contrast of the image to make easier the visualization of the maximums and minimums.

In addition, there is a tool to measure distances in pixels in the digital image. For instance, when we measure the width of the central maximum of a square aperture diffraction, the front panel looks like Figure 3.

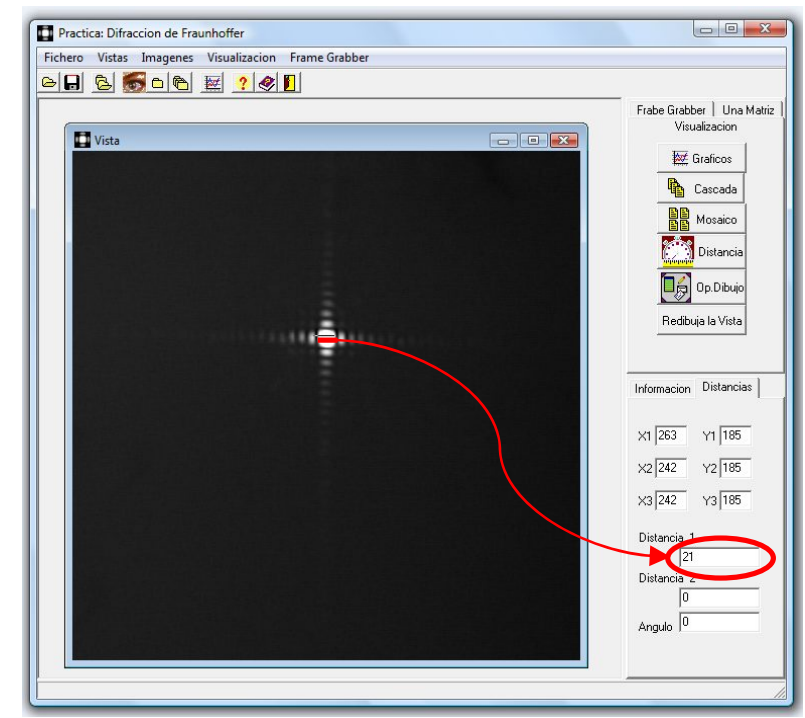

Figure 3: Software front panel used to measure distances in pixels. In particular, here we are measuring the width of the central maximum for the diffraction of a square aperture (21 pixels). 
Also, the program lets the user measure the angle between two segments. This is a useful tool to analyze angular properties of apertures (for instance, the angles of a triangle).

The cross sections of the diffraction pattern in $\mathrm{X}$ and $\mathrm{Y}$ axes can be also visualized (see Figure 4) or a datasheet with all the values can be obtained.

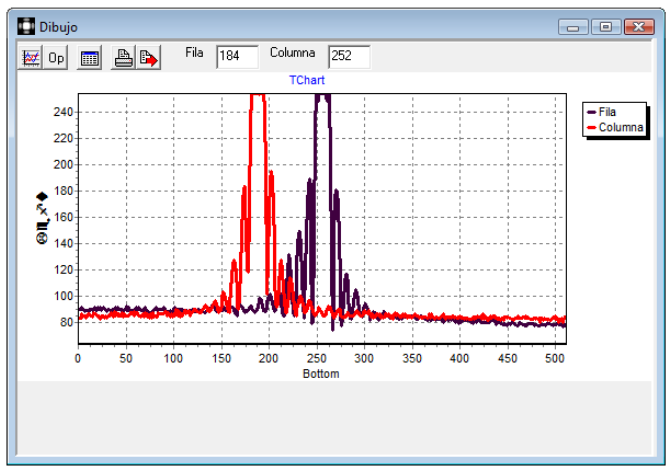

Figure 4: Cross sections of (in magenta-right peak) X and (in red-left peak) Y axes, when the mouse is in the center of the pattern showed in Figure 3.

Finally, students use a module to simulate diffraction patterns of different apertures. With that purpose, the user introduces the features of the aperture in a window like Figure 5. They can control several parameters: shape of the basic aperture (rectangle, cercle or slit), dimensions of the aperture (radius of the cercle, widths of the rectangle or thickness of the slit), number of identical apertures (single, double, one or two dimensional gratting), wavelength, focal length of the imaging lens, image size and pixel size (calibrated conversion factor).

Therefore, the module allows them to reproduce the images obtained experimentally, and generate new ones by changing certain parameters. These simulations lead students to analyze a large variety of apertures and their diffraction pattern, as well as understanding how each parameter has an effect on the diffraction pattern. For instance, in our setup all the apertures are illuminated with a He-Ne laser, so all the diffraction patterns are obtained with the same wavelength. However, using the simulation, students can see what happens if the aperture is illuminated by a shorter or a larger wavelength, understanding the role of that parameter in the diffraction formula. In the same way, a study of the effect of the focal length on the intensity distribution can be conducted.

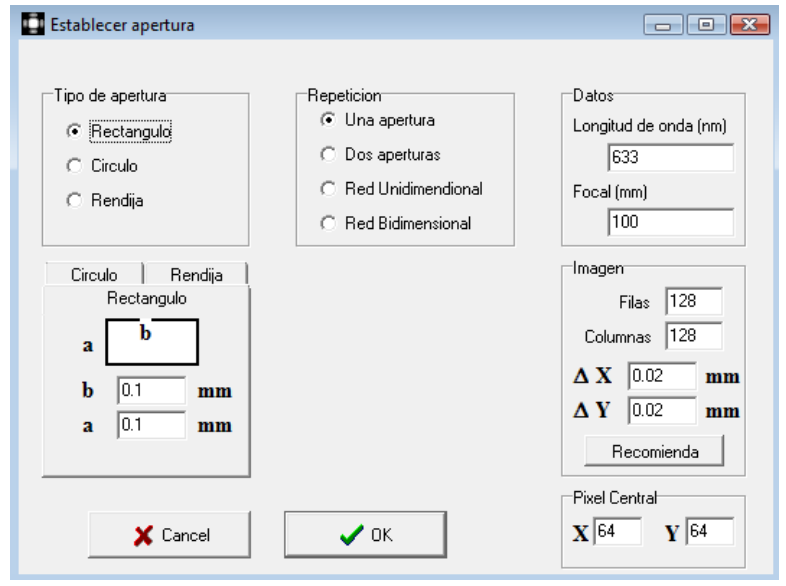

Figure 5: Window where the user specifies the features of the aperture to simulate its diffraction. 


\section{RESULTS}

\subsection{Experimental results}

Figure 6 shows a set of pictures acquired by students during laboratory sessions of this course 2011-2012.

By looking to the diffraction of a single slit they can visualize the sinc function in one dimension (Figure 6(a)-(b)). Also, they notice the effect of the width of a single slit on the Fourier Transform. Then, they analyze the effect of adding another slit (Figure 6(c)-(d)). They see how the interference term of two slits is reflected in secondary maxima and the modulating function is still the diffraction pattern of a single slit. These diffraction patterns allow them to understand the dependence on the width and separation between two slits. After that, they observe the Fourier Transform of 1dimensional grating (Figure 6(e)-(f)), noticing how the number of slits per millimeter changes the distance between maximums.

Next, fundamental polygons apertures are studied. They observe the diffraction of a square aperture, where two sinc functions with the same frequency are distributed in the $\mathrm{X}$ and $\mathrm{Y}$ axes (Figure 6(g)). They deduce the distribution of the two sincs functions for a rectangular aperture (Figure 6(h)). The next figure to analyze is a triangular aperture [9] (Figure 6(i)). Students, by using the specific tool in the software, can determine the angles of the actual aperture.

Then, they visualize the Airy disk (Figure 6(j)), and we ask them to determine the radius of the aperture during the laboratory session. This simple calculation does not consume too much time during the session, but it is really helpful for them to understand the intrinsic relation between the diffraction pattern and the size of the obstacle. That calculation leads to explain applications of particle characterization by analyzing the Fraunhofer diffraction [10-12].

Moreover, students analyze 2-D gratings. There, they can visualize the convolution theorem [3]. This is a difficult concept to understand just with theory lessons. The theorem states the Fourier Transform of a direct product is the convolution of the Fourier Transforms. On the other hand, the Fourier Transform of a convolution is the direct product of the individual Fourier Transforms.

$$
\begin{aligned}
& \Im[f \cdot g]=\Im[f] \otimes \Im[g] \\
& \Im[f \otimes g]=\Im[f] \cdot \Im[g]
\end{aligned}
$$

In particular, they observe the intensity distribution of 2-dimensional square grating of elemental apertures limited by a circular window, where the elemental apertures are squares (see Figure 6(k)), or circles (see Figure 6(1)). In addition, they get used to the following notation to express complex apertures described by the convolution theorem. Here we show the Fourier Transform of a square grating of circles, limited by a circular aperture:

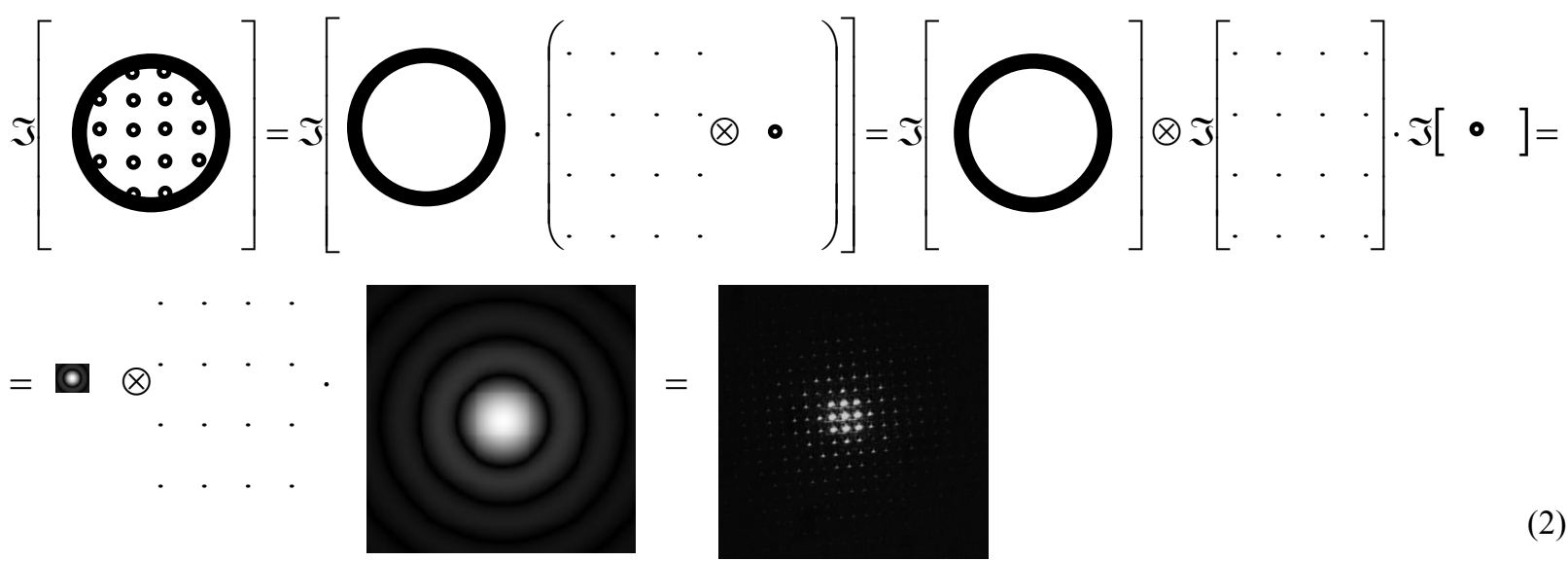


(a)

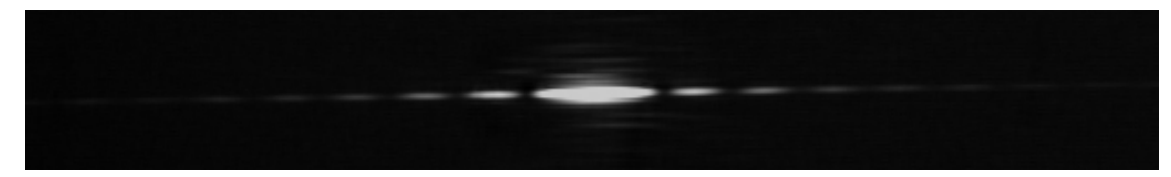

(b)

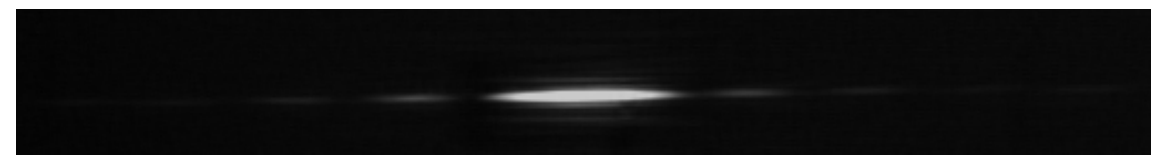

(c)

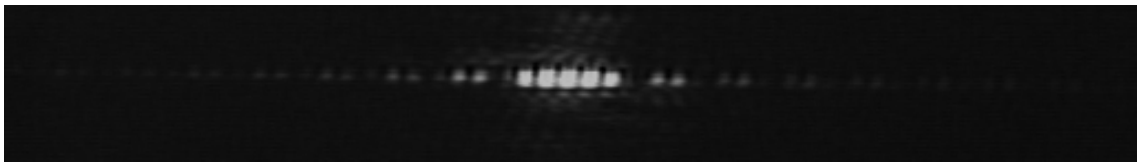

(d)

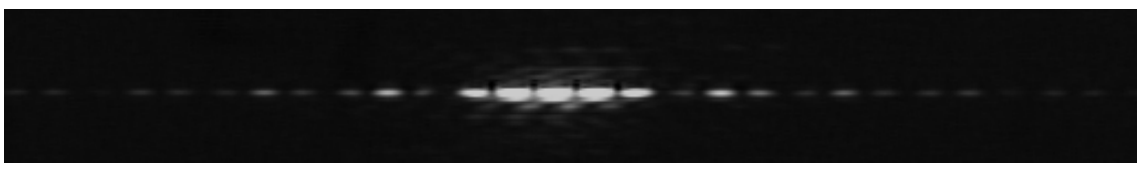

(e)

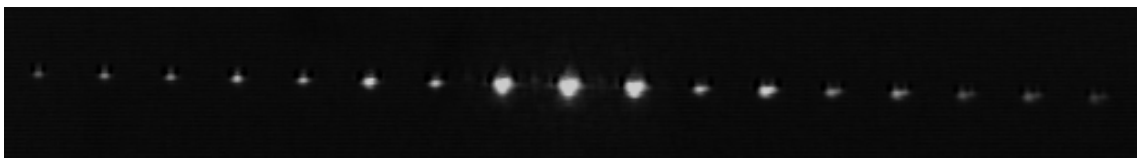

(f)
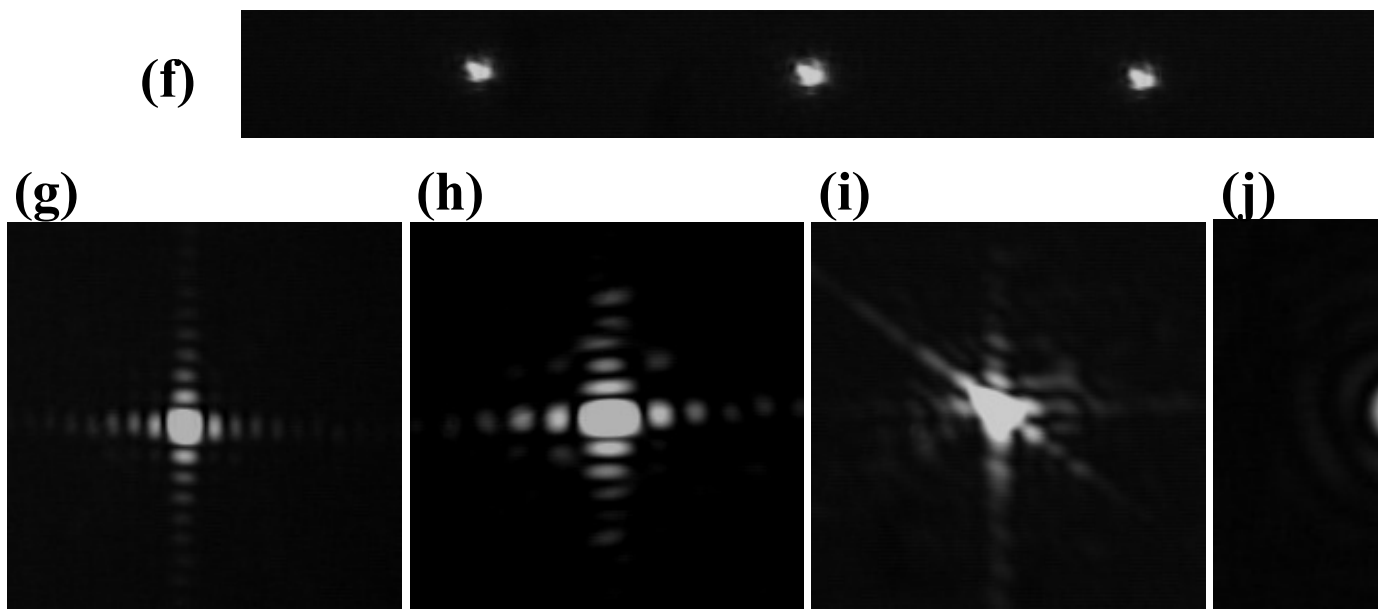

(h)

(i)

(i)
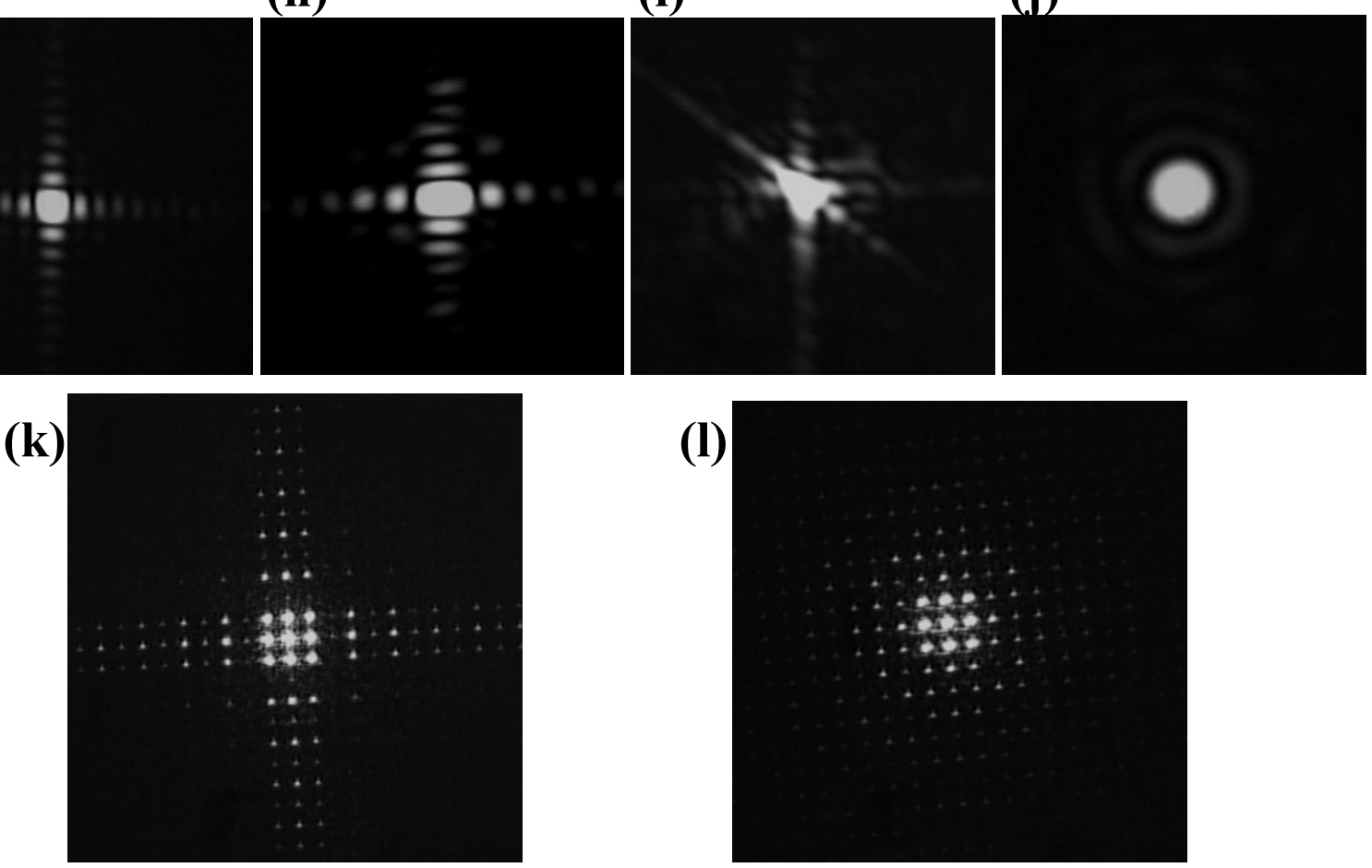

(l)

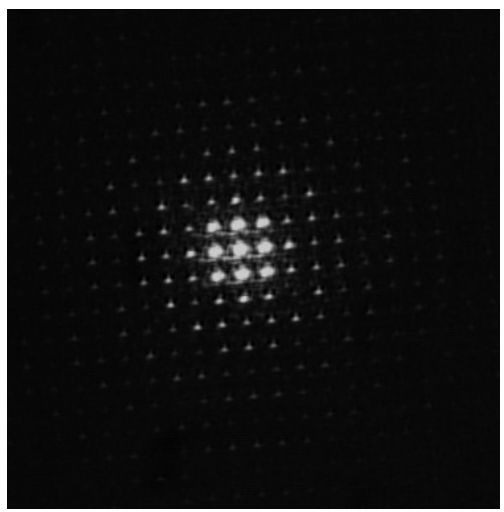

Figure 6: Images acquired by students of the different diffraction patterns observed in the laboratory. The obstacles are: (a) a single slit, (b) a narrower slit, (c) double slit, (d) closer double narrower slit, (e) 1-D grating, (f) 1-D grating with closer slits, (g) square aperture, (h) rectangular aperture, (i) triangular aperture, (j) circular aperture, (k) 2-D square grating of square apertures limited by a circular window, and (1) 2-dimensional square grating of circular apertures limited by a circular window. 


\subsection{Simulation results}

The simulation module allows reproducing new configurations respect to the ones analyzed in the laboratory. For instance, they can analyze the diffraction pattern of a circular aperture illuminated with different wavelengths, and not just with the one given by a He-Ne laser. In the plot of Figure 7(a), we can appreciate that the radius of the Airy disk increases linearly as the wavelength gets larger. Moreover, in Figure 7(b), we observe that the radius of the disk is directly proportional to the focal length of the imaging lens. Therefore, they are verifying by simulations the formula which gives the position of the first minimum of diffraction for a circular aperture:

$$
\rho=1.22 \frac{\lambda f}{2 R},
$$

where $\rho$ is the position of the first minimum in the diffraction pattern (radius of the Airy disk), $f$ is the focal imaging lens, $\lambda$ is the wavelength and $R$ is the radius of the circular aperture.
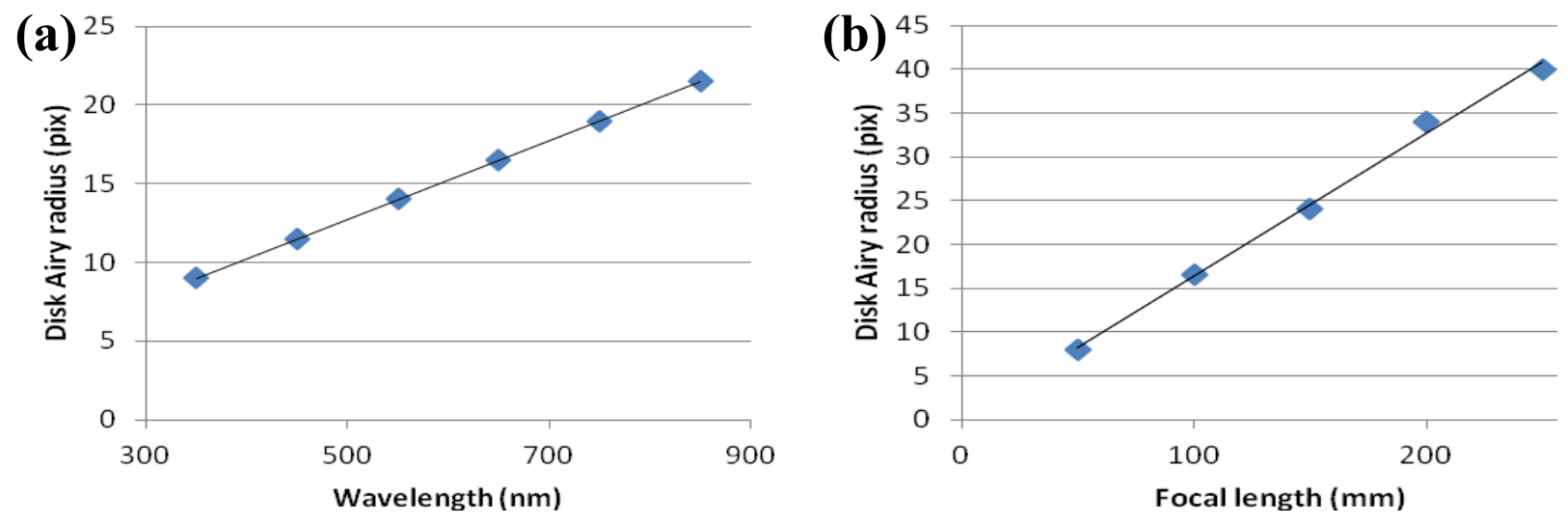

Figure 7: Study, by simulation, of the radius of the Airy disk, when we change (a) the wavelength, or (b) the focal length of the imaging lens. The other parameters are kept constant at the values used in the laboratory.

In order to fully understand the convolution theorem, the students can simulate additional diffraction patterns apart from the ones visualized during the laboratory session. For instance, in Figure 8, they have simulated the Fourier Transform of the convolution of one dimensional comb function with a circular aperture.

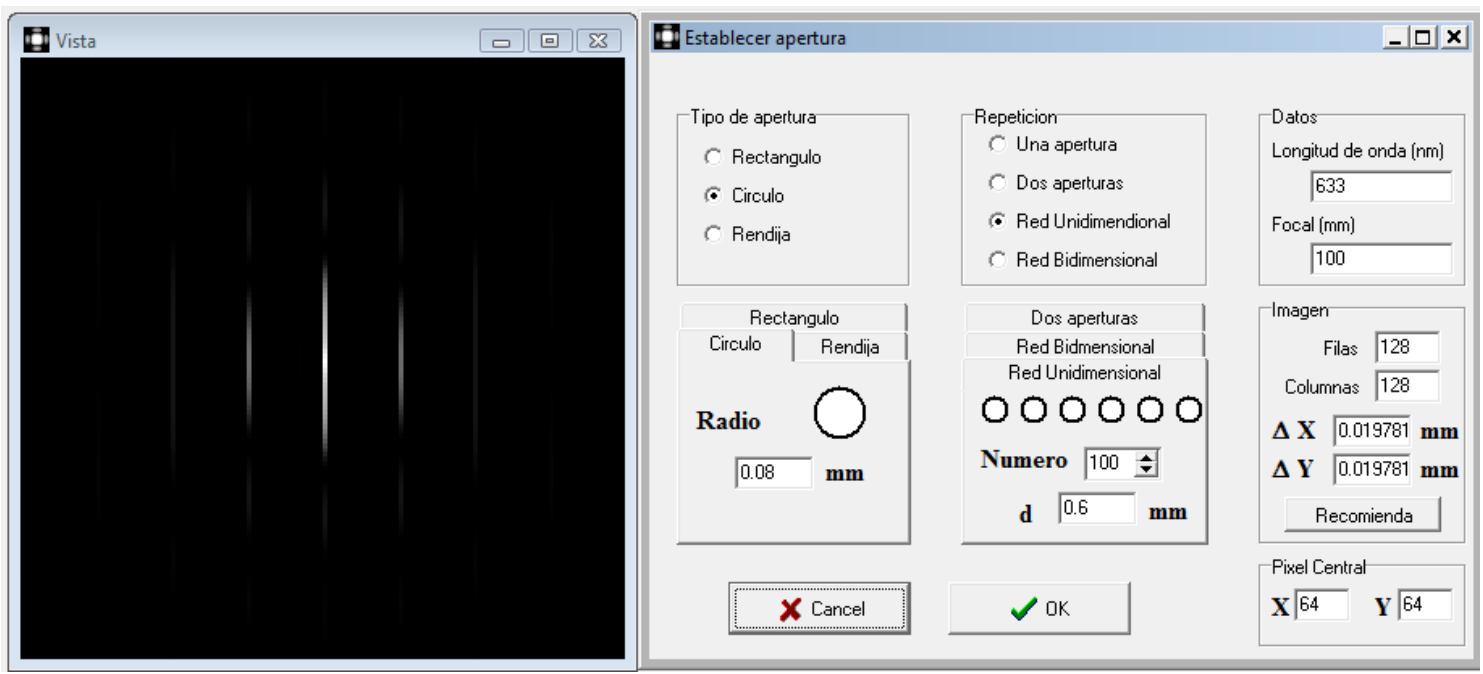

Figure 8: Simulated diffraction pattern of an object based on a 1 dimensional grating of 100 circular apertures of radius $0.08 \mathrm{~mm}$. The distance between elemental apertures is $0.6 \mathrm{~mm}$. The wavelength is $633 \mathrm{~nm}$, and the focal lens is $100 \mathrm{~mm}$. 


\section{EVALUATION}

The evaluation of Applied Optics course is adapted by the regulation of Bologna process [13]. This process has several goals. In relation to the evaluation process, it pretends that the evaluation must take into account more and more precise elements of the student's work. Thus, it must take into account exams, home works, experimental works, oral presentations, etc. That is to say, a continuous evaluation is intended. The laboratory work of that course has different evaluation activities which assess the objectives and skills acquired by the student.

In particular, it includes the following academic methodologies. First of all, the assistance to the laboratory sessions is required in order to be evaluated. The students receive a questionnaire about basic concepts learned during the laboratory session. Individually, students will answer and hand in it one week later. In that way, students have still the concepts fresh in their mind, helping to consolidate them. After realizing the three laboratory sessions, they choose one of them and, in team work, they prepare a comprehensive report. In the end of the course, the team will have an oral defense of the report, in order to prove the participation of all the students, the correct understanding and assimilation of the experiment, and to discuss the most relevant results of the report. Moreover, some process assignments, including problems and simulations using Matlab, are asked to students. Finally, there is a final written exam of the course, where one question about laboratory will be asked. Table 1 shows the weights that each task has on the global grade. It shows that $50 \%$ of the global grade comes from practical tasks made by the students.

\begin{tabular}{|c|c|}
\hline Task & Percentage in global grade \\
\hline Matlab simulations & $15 \%$ \\
\hline Home work exercises & $10 \%$ \\
\hline 3 questionnaires & $10 \%$ \\
\hline Lab report + oral presentation & $15 \%$ \\
\hline Final exam & $50 \%$ \\
\hline
\end{tabular}

Table 1: Evaluation of the subject.

\section{FINAL COMMENTS}

This work describes a training based on experimental and simulated work for undergraduate students to complement the theory lectures of Fraunhofer diffraction.

On the one hand, laboratory training is essential in the syllabus of a scientific student since it allows applying the theoretical knowledge to the real physical world. Moreover, learners acquire skills in experimental methodology, they get used to common material in an optical laboratory (lenses, spatial filter, polarizer, laser) and to analyze experimental data.

On the other hand, simulations helps to consolidate main concepts since they can reproduce new configurations different from the one used in the laboratory.

Furthermore, graduate students help during the execution of the experiment as laboratory teaching assistant, being their first contact with teaching.

\section{ACKNOWLEDGEMENTS}

We acknowledge financial support from Spanish Ministerio de Ciencia e Innovación (FIS2009-13955-C02-01) and Generalitat de Catalunya (2011FI_B2 00140). 


\section{REFERENCES}

[1] Hecht, E., [Optics], Addison-Wesley, 4th edition, Massachusetts (2001).

[2] Casas, J., [Óptica], Librería Pons, 7th edition, Zaragoza, Spain (1994).

[3] Goodman, J. W., [Introduction to Fourier Optics], Mc Graw - Hill, 2nd Edition (1997).

[4] Reynolds, G. O., Develis, J. B., Parrent, G. B. and Thompson, B. J., [The New Physical Optics Notebook: Tutorials in Fourier Optics], SPIE Optical Engineering Press, Washington (1989).

[5] Vanderlugt, A., [Optical Signal Processing], John Wiley, New York (1992).

[6] Collier, R. J., Burckhardt, C. B., Lin and L. H., [Optical Holography], Academic Press Inc., New York (1971).

[7] Centro de Holografía de Alicante, [Holografía], Colección Universidad-Caja de Ahorros de Alicante, Secretariado de Publicaciones de la Universidad de Alicante, Alicante (1985).

[8] Yzuel, M. J., Escalera, J. C., Lizana, A., Espínola, M. and Campos, J., "A laboratory of image processing and holography for physics students," ETOP Conference Proceedings, 214-223 (2007).

[9] Smith, R. and Marsh, J., "Diffraction patterns of simple apertures," J. Opt. Soc. Am. 64, 798-803 (1974).

[10] Cornillault, J., "Particle Size Analyzer," Appl. Opt. 11, 265-265 (1972).

[11] Heuer, M. and Leschonski, K., "Results Obtained with a New Instrument for the Measurement of particle size distributions from diffraction patterns," Part. Part. Syst. Charact. (2), 7-13 (1985)

[12] Lunven, M., Gentien, P., Kononen, K., Le Gall, E. and Daniélou, M. M., "In situ video and diffraction analysis of marine particles," Estuarine, Coastal and Shelf Science, Volume 57, Issues 5-6, 1127-1137(2003).

[13] http://www.uab.es/bologna 